\title{
DIÁRIO DE USO DIGITAL - MAPEANDO AS IMPRESSÕES E AVALIAÇÕES DOS USUÁRIOS.
}

DIARY OF DIGITAL USE - MAPPING THE IMPRESSIONS AND REVIEWS OF USERS.

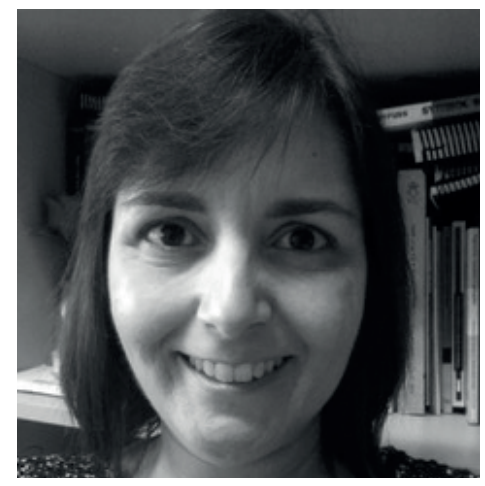

Cláudia Mont'Alvão, D.Sc LEUI, PUC-Rlo

e-mail: cmontalvao@puc-rio.br

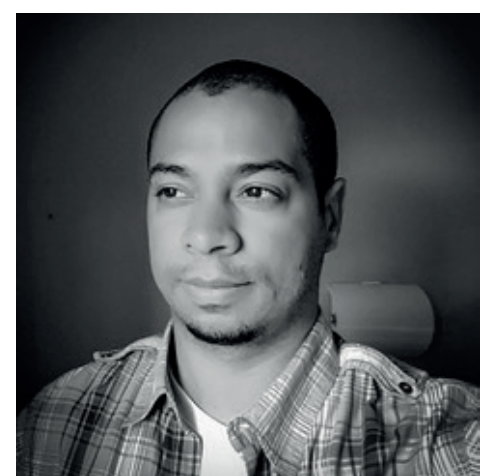

Flávio Nazário, M.Sc

LEUI, PUC-Rlo

e-mail: info@flavionazario.com 


\section{RESUMO}

O Diário de uso digital é um método de acompanhamento prolongado que meIhora significativamente os níveis de envolvimento dos usuários devido à sua praticidade, mobilidade e espontaneidade na permuta de informação entre os participantes e os pesquisadores do estudo. O método auxilia a compreensão contextual de comportamentos dos participantes ao longo do tempo, com o mínimo de impacto ou interferência no cotidiano das pessoas.

O artigo aborda o planejamento e a utilização de Diários de uso para recolher dados qualitativos e quantitativos das impressões e expectativas dos participantes durante um período de tempo, que variou de alguns dias a um mês de utilização do aplicativo (app) Duolingo. Durante o período definido, os participantes foram convidados a manter um diário de uso digital através do app Experience Fellow ${ }^{\circledast}$, instalado nos seus respectivos smartphones.

Os estudos envolvendo este método exigem tempo e dedicação dos participantes e um elevado grau de meticulosidade dos pesquisadores envolvidos. Entretanto, o trabalho de equilibrar a utilização constante do app Duolingo com os registros no Diário digital dos envolvidos costuma ser um dos maiores desafios do método na busca por descobertas relevantes para o resultado da pesquisa.

\section{PALAVRAS-CHAVE}

Diário de Uso; Duolingo; Experiência do Usuário; Jornada do Usuário; Sonda Cultural.

\section{ABSTRACT}

The Diary Study is an extended follow-up method that significantly improves the levels of participation of users due to its practicality, mobility, and spontaneity of the information's exchange between the study's participants and researchers. The method assists the contextual understanding of participants' behaviors over time, with minimal impact or interference in people's daily lives.

This article discusses the planning and use of a Digital Diary Study to collect qualitative data about participants' impressions, and expectations over a period ranging from a few days to a month of using the Duolingo app. During the defined period, participants were invited to keep a digital diary through the Experience Fellow ${ }^{\circledR}$ app, installed on their smartphones.

Studies involving this method requires time and dedication of the volunteers and a high degree of meticulousness from the researchers involved. However, the nuances of balancing the constant use of the Duolingo app and the entries in the diaries are usually one of the technique's major challenges in finding relevant insights for the research result. 


\section{KEYWORDS}

Diary Studies; Duolingo; User Experience; User Journeys; Design Probe.

\section{INTRODUÇÃO - INVESTIGANDO HÁBITOS ATRAVÉS DE DIÁRIOS}

O Diário de uso (Diary Study), também conhecido como Sonda ou Sondagem Cultural (Cultural Probe ou Design Probe) é um método onde as pessoas registram em um local, geralmente um diário, seu progresso e/ou suas experiências com um produto/serviço durante vários dias, semanas ou meses como sugerido por Alaszewski (2006) e Lazar et al. (2010).

Sobre as origens do método, Lazar et al. (2010) explica que o diário de uso na área de IHC foi adaptado a partir de outras áreas de estudo, principalmente pesquisas de Sociologia e História. Na Sociologia, diários são usados para entender como é a experiência individual. Outros campos de estudo, como a área de saúde, por exemplo, utilizam o diário como método complementar para acompanhar pacientes em recuperação ou que estão participando de testes de medicação.

Mattelmäki (2006) traça um panorama histórico do uso do método reforçando que as mudanças no campo do Design também afetaram as técnicas de coleta de dados centradas no usuário. Desde a década de 1990, métodos experimentais têm sido agregados ao campo do Design centrado no usuário. A sondagem (probe ou probing) está entre essas novas técnicas empregadas, atendendo às demandas de experimentação e interação com o usuário. A sondagem é estabelecida no campo do Design com a finalidade de encontrar e delinear soluções alternativas, destinando-se a apoiar tanto os designers como os usuários no mapeamento de suas experiências. As atribuições da sondagem são, ao mesmo tempo, descritivas e exploratórias, o que possibilita a abertura e espaço para a interpretação, avaliação e a possibilidade de um resultado surpreendente ou inesperado.

O formato mais comum de um diário de uso é o de tempo. Lazar et al. (2010) explica que o diário se concentra em observar os registros no tempo em que os indivíduos utilizavam o produto/serviço em diferentes atividades. A principal diferença entre um diário geral e um diário de tempo é que as anotações do diário geral podem estar em uma base frequente ou não-temporal, enquanto que o diário de tempo tem um foco exatamente, como o próprio nome diz, no tempo, pois grande parte da pesquisa em IHC concentra-se em quanto tempo utilizamos algum aplicativo, produto/serviço ou quanto tempo perdemos devido a frustrações ou alternâncias de tarefas. Por conta disso, diários de tempo são muitas vezes o tipo predominante em pesquisas em IHC, como este que é descrito neste artigo. 
Murphy (2006) destaca que as sondas culturais são ferramentas adicionais que apresentam um conjunto de pontos fortes e fracos. Por exemplo, a confiabilidade dos dados pode ser melhorada quando os usuários são especialistas em suas próprias vidas. Desse modo as sondas culturais fornecem a oportunidade dos usuários documentarem suas vidas em seu próprio contexto, com interferência mínima do designer. As sondas também fornecem a oportunidade de fazer uma triangulação com outro conjunto de dados independentes como grupos de foco, testes de usabilidade ou resultados de levantamento e assim melhorar a confiabilidade dos resultados.

O Diário de uso relatado aqui foi uma das técnicas utilizadas na pesquisa realizada por Nazário e Mont'Alvão (2017) que investigou as motivações e as interpretações dos usuários ao utilizarem o app Duolingo para a construção de conhecimento do idioma inglês, aplicativo muito conhecido na categoria de Aprendizagem Móvel (M-Leaning).

A técnica foi escolhida, pois permite um bom entendimento da rotina e das suas possibilidades de uso. Além disso, o Diário de Uso representa uma alternativa, na tentativa de minimizar a possível influência do observador sobre a pessoa observada, auxiliando na descoberta de 'como' e 'onde' os usuários utilizam o produto analisado no cotidiano real.

Desta forma, o diário é um instrumento típico de sondagem, com foco em rotinas e sentimentos do usuário, contendo pensamentos, atividades de registro e fenômenos da vida cotidiana e privada. Vale ressaltar que o diário tem um foco estabelecido com e para o usuário. Neste sentido, cada registro dos participantes é único e reforça a ênfase na importância dos dados qualitativos da pesquisa.

\section{CONDUZINDO A TÉCNICA METODOLÓGICA DE FORMA CONSISTENTE}

Para aplicar e conduzir a aplicação da técnica de diário de uso é necessário planejar algumas etapas e processos que garantem a confiabilidade da coleta de dados. Mattelmäki (2006) descreve quatro fases principais para isso.

Na primeira etapa, intitulada Sintonizando (tuning in), é definido o objetivo da coleta de dados, que precisa ser escrito de forma clara, atrelado ao tipo de investigação que se pretende fazer. Os objetivos devem tratar de questões ligadas ao cotidiano do usuário e sua possível experiência de uso e não do produto desejado. Importante explicar ao usuário as razões da existência do diário de uso ou sondas culturais e para quais fins elas serão projetadas inicialmente. Além disso, os pesquisadores envolvidos podem documentar suas opiniões do 
que esperam tentar descobrir com o método. Posteriormente esses dados podem ser cruzados com os resultados finais.

A segunda etapa é o Recrutamento de público-alvo (reaching out to the target group). Deve-se considerar dados demográficos como idade, gênero e estilo de vida, além do local onde vivem os participantes para seleção da amostra estudada, sempre alinhados com os objetivos da pesquisa. Quanto ao tamanho do grupo, tanto Mattelmäki (2006), quanto Lazar et al. (2010) e Goodman (2012) consideram que o grupo deve ser pequeno, de 5 a 10 participantes. Porém esses participantes devem ter um perfil significativo, mais "independente" ou "envolvido", pois boa parte das atividades relacionadas à aplicação da técnica requerem disciplina e motivação dos participantes. Deve ser também considerada a logística de distribuição de materiais (se o diário for físico), das entrevistas e da interpretação de resultados ao montar o grupo final de participantes. $\mathrm{Na}$ terceira etapa projeta-se o diário de uso/sonda cultural (designing the probes). O diário pode ser de papel ou eletrônico, sendo possível a utilização de materiais disponíveis no comércio, cadernetas ou ainda, se produzir pequenos livretos semelhantes a documentos. A forma eletrônica pode ser uma opção interessante, caso os participantes tenham acesso e/ou estejam preparados para o uso de equipamentos de informática ou dispositivos computacionais.

O período de aplicação da técnica pode ser variado. Por isso, é importante realizar contatos durante a aplicação, principalmente, no caso de períodos prolongados, de forma a manter os relatores entusiasmados com a atividade. Revisar as orientações de preenchimento, além de tirar dúvidas durante o período também podem ser interpretadas como ações motivadoras.

Para se aplicar efetivamente a técnica, duas características precisam ser consideradas: projetar o diário com o mínimo de 'autossuficiência', se necessário com explicações inclusas para permitir que os participantes o utilizem sem dificuldades e efetuar testes piloto com pessoas de público similar ao Diário de uso final, para que se possa fazer ajustes na compreensão e no manuseio do material.

A última etapa (follow-up of the probe material), consiste em se fazer uma entrevista ou debriefing com os participantes 'diaristas'. Deve-se começar a entrevista relembrando o objetivo da pesquisa e reafirmando o caráter de anonimato dos dados que foram recolhidos no diário de uso e os relatos que serão expostos na entrevista. É recomendado que seja feita uma análise prévia do material antes da entrevista, para que as perguntas estejam relacionadas como que já é conhecido, tornando a entrevista mais fluida para ambas as partes. A entrevista deve ser direcionada às histórias dos participantes, novas oportunidades de 
design e motivações, e não simplesmente a "coleta de fatos" do entrevistado. Contudo, ao final das etapas, o foco dos Diários de uso não está na análise dos dados de forma precisa e no controle cuidadoso da sua aplicação da técnica, mas sim, em nas implicações culturais do projeto e no descobrimento de novos caminhos para um eventual novo serviço/produto ou suas melhorias. É preciso abraçar a subjetividade e a personalidade das pessoas, já que o que se procura é a inspiração e a motivação dos usuários, em relação a utilização do serviço/ produto e não a informação 'pura' na aplicação da técnica.

\section{PROCEDIMENTOS UTILIZADOS NO DIÁRIO DE USO}

Como dito anteriormente, este artigo apresenta não somente a fundamentação teórica da técnica, mas também a aplicação e discussão de sua utilização. O objetivo da aplicação dos Diários na pesquisa era verificar se a hipótese de uso de um aplicativo de ensino de línguas, como o Duolingo, durante o cotidiano, traria benefícios para aprendizagem dos participantes, já que a utilização do smartphone pelo usuário facilitaria seu acesso à prática de exercícios em qualquer ocasião disponível que surgisse.

Com o objetivo definido foram necessárias três etapas para o recrutamento, a primeira delas foram quatro entrevistas com professores para entender o atual cenário do ensino e aprendizagem aliado a tecnologias diversas. Através da análise desse insumo, dois questionários de triagem foram produzidos: o primeiro traçava o perfil demográfico e hábitos de uso de tecnologia dos respondentes e o segundo, recolhia informações sobre formas de estudo do idioma e a experiência com língua estrangeira.

Desta forma, emergiram dois perfis predominantes na pesquisa: o primeiro, tinha interesse no tema e se mostrava solícito para utilizar a aprendizagem móvel como suporte válido para aprimorar seus estudos, ainda iniciais em um outro idioma. O segundo, representando boa parte dos respondentes, tinham como principais características a curiosidade por tecnologia, o conhecimento de intermediário para avançado da língua inglesa e/ou outro idioma e o interesse pela forma de interação das aplicações de aprendizagem móvel, apesar de apresentarem dúvidas sobre sua efetividade de aprendizado pelo smartphone. Foram convidados 15 participantes através dos questionários online em momentos distintos. Seguindo premissas de confidencialidade, cada participante teve seu nome substituído por uma sigla de identificação. A amostra selecionada foi de conveniência e a composição final ficou com $60 \%$ do sexo feminino e $40 \%$ do sexo masculino e faixas etárias que variavam de 19 a 33 anos. Todos 
apresentavam pelo menos 1 hora de tempo livre para participar da pesquisa diariamente e eram estudantes universitários de graduação ou pós-graduação. A maioria dos participantes eram usuários do Sistema Operacional Android, apenas 2 participantes utilizavam outros sistemas: 1 participante utilizava iOS e 1 Windowsphone. A tabela 1, a seguir, apresenta um resumo das características dos voluntários do Diário de uso. Um dos fatores que também pesou na decisão sobre a escolha dos participantes foi nenhum ou baixo conhecimento de M-Learning. A intenção foi garantir o teor de novidade ao inserir-se o novo hábito na rotina do usuário, de forma que fosse possível verificar como cada um 'encaixaria' a utilização dos apps em sua rotina.

Tabela 1: Diário de uso - perfil dos participantes

\begin{tabular}{|c|c|c|c|c|c|}
\hline & Gênero & Idade & Curso idioma & $\begin{array}{c}\text { Anos de } \\
\text { estudo }\end{array}$ & $\begin{array}{l}\text { Conhece } \\
\text { Duolingo }\end{array}$ \\
\hline P1 & Fem & 23 & - & $\begin{array}{c}\text { Mais de } 5 \\
\text { anos }\end{array}$ & Sim \\
\hline P2 & Fem. & 21 & CNA e Ibeu & 3 a 4 anos & Não \\
\hline P3 & Masc & 24 & $\begin{array}{l}\text { Cultura } \\
\text { Inglesa }\end{array}$ & $\begin{array}{c}\text { Mais de } 5 \\
\text { anos }\end{array}$ & Sim \\
\hline P4 & Masc & 23 & $\begin{array}{l}\text { Cultura } \\
\text { Inglesa }\end{array}$ & 3 anos & Não \\
\hline P5 & Fem. & 20 & $\begin{array}{l}\text { Cultura } \\
\text { Inglesa }\end{array}$ & 3 anos & Não \\
\hline P6 & Masc & 22 & Ibeu & $\begin{array}{c}\text { Mais de } 5 \\
\text { anos }\end{array}$ & Não \\
\hline P7 & Masc. & 21 & $\begin{array}{l}\text { CCAA e } \\
\text { Cultura } \\
\text { Inglesa }\end{array}$ & $\begin{array}{c}\text { Mais de } 5 \\
\text { anos }\end{array}$ & Não \\
\hline P8 & Fem. & 32 & - & 1 ano & Não \\
\hline P9 & Fem. & 21 & Yes! & 4 anos & Sim \\
\hline P10 & Fem. & 19 & $\begin{array}{l}\text { Cultura } \\
\text { Inglesa }\end{array}$ & $\begin{array}{c}\text { Mais de } 5 \\
\text { anos }\end{array}$ & Não \\
\hline P11 & Masc. & 33 & Oxford & 2 a 3 anos & Sim \\
\hline P12 & Fem. & 20 & $\begin{array}{l}\text { CNA e Cul- } \\
\text { tura Inglesa }\end{array}$ & 1 a 2 anos & Sim \\
\hline P13 & Masc. & 20 & $\mathrm{CNA}$ & 1 a 2 anos & Sim \\
\hline P14 & Fem. & 23 & - & - & Não \\
\hline P15 & Fem. & 19 & Brasas e Fisk & 3 a 4 anos & Não \\
\hline
\end{tabular}

Com o perfil do público participante definido, a segunda etapa consistia-se em 
definir, configurar ou projetar o Diário de Uso. Optou-se aqui em se fazer o acompanhamento por intermédio da plataforma Experience Fellow ${ }^{\circledR}$. A plataforma consiste de um aplicativo nativo (Android e iOS) no smartphone dos participantes e de um website onde os pesquisadores podem acompanhar as anotações dos voluntários. Apenas um dos participantes não conseguiu utilizar o app Experience Fellow ${ }^{\circledR}$ por ter um dispositivo Windowsphone. Portanto, para essa pessoa um diário de papel foi confeccionado nos moldes e com informações iguais às do diário digital.

Na Figura 1 é possível verificar como se dá o fluxo de anotações de experiências pelo participante. Após acessar o app já instalado no seu dispositivo e ler as instruções na tela azul, é necessário criar uma nova anotação (touchpoint) na tela seguinte. Dentro da seção de touchpoint, o participante preenche qual foi seu nível de satisfação ao utilizar o Duolingo (ícones de humor) e por fim, pode optar por adicionar mais detalhes, anexar imagens ou gravar um vídeo do que ocorreu após o uso. Feito isso, o participante faz o upload das anotações do dia, tornando-as visíveis na plataforma web acompanhada pelo pesquisador.

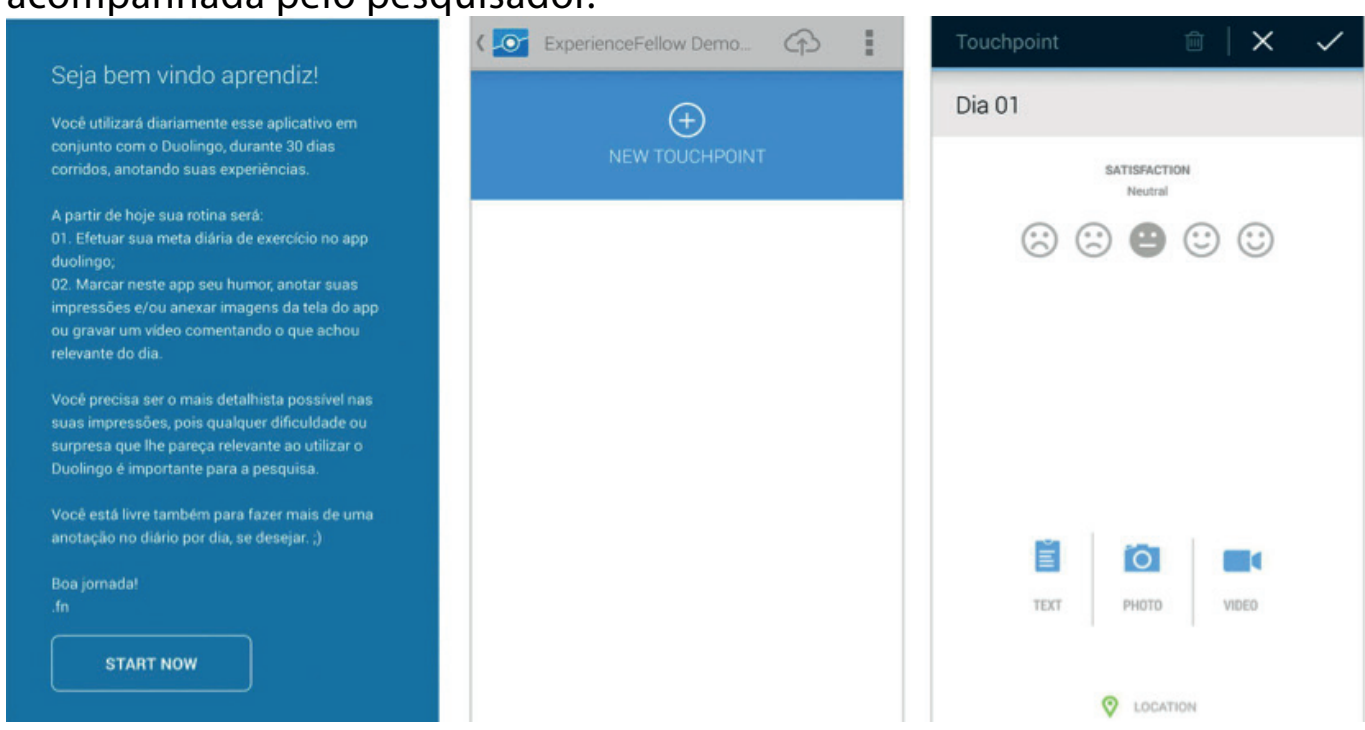

Figura 1: Experience Fellow - fluxo de anotações

Fonte: App Android Experience Fellow, 2016.

A plataforma Experience Fellow ${ }^{\oplus}$ foi a escolhida para acompanhamento, pois seu website disponibiliza uma série de possibilidades de visualizações, como as anotações dos participantes em forma de jornada (Figura 2). Além da possibilidade de exportar todas informações registradas pelos participantes em tabelas. 


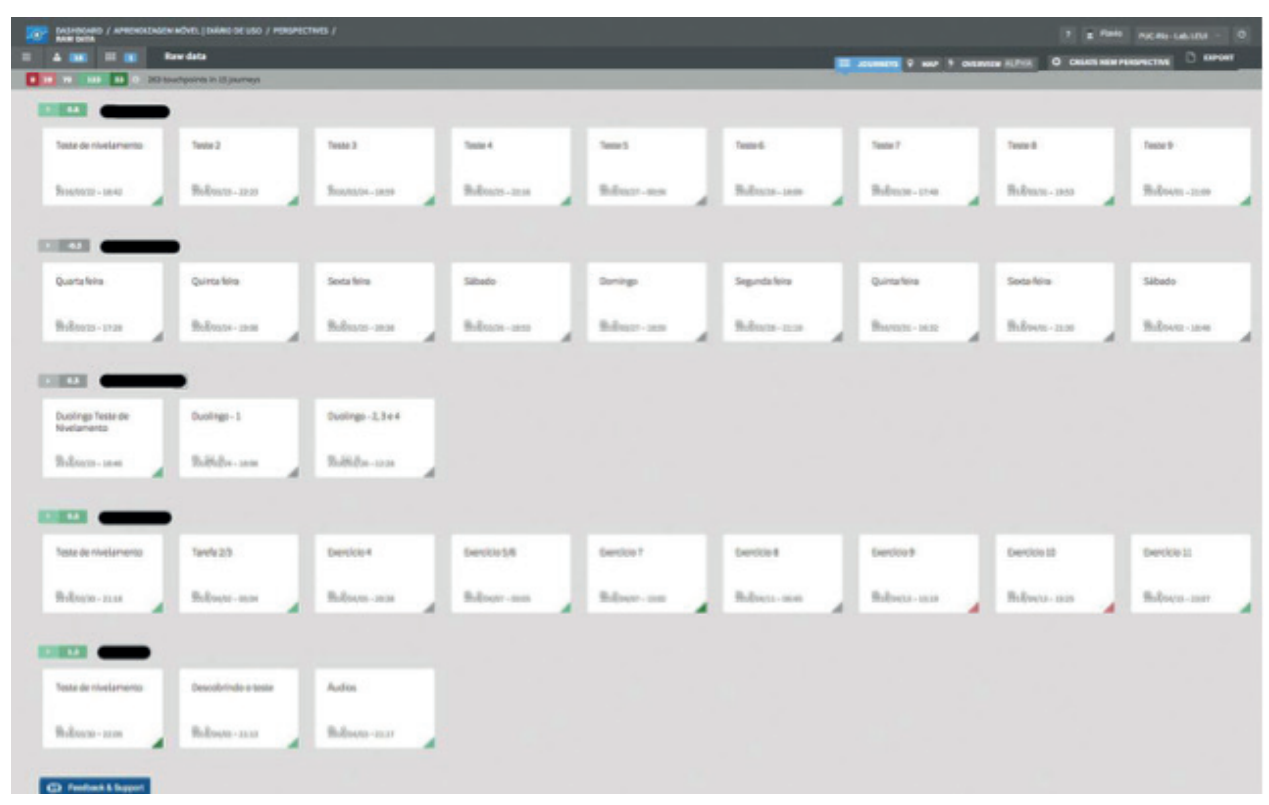

Figura 2: Experience Fellow - jornada dos participantes

Fonte: Website Experience Fellow, 2016.

Como última etapa do processo, similar a uma entrevista, o debriefing foi empregado como um guia de tópicos gerais para validar ou não a hipótese levantada. Seja após a desistência de alguns participantes, antes de completar 30 dias de uso ou ao término da quantidade estipulada de dias de uso, os participantes foram convidados a expor suas interpretações e/ou motivações ao utilizar o Duolingo, assim como questões relacionadas a aprendizagem móvel de acordo com o objetivo específico estabelecido pela pesquisa. O debriefing abordava em torno de 10 tópicos que investigavam informações relacionadas aos conceitos de mobilidade e aprendizagem, tais como, a forma como o usuário fazia uso de ambos apps simultaneamente e qual era sua impressão sobre poder estudar em qualquer lugar (mobilidade física). Os tópicos consistiam em: a) Suas principais dificuldades com o Duolingo, b) suas impressões utilizando seu dispositivo como plataforma de estudo complementar; c) se o Duolingo auxiliou de fato na adição de conhecimento (mobilidade conceitual); d) se o app Duolingo estimulou o interesse em compartilhar com pessoas ou amigos os estudos (mobilidade sociointeracional); e) se o participante acredita que é possível aprender algo exclusivamente pelo seu smartphone.

Na ocasião do debriefing, o pesquisador solicitou a gravação dos áudios das conversas através de um smartphone para análise detalhada posterior.

\subsection{PROCESSO E APLICAÇÃO DOS DIÁRIOS DE USO}

Para a aplicação da técnica foram efetuados pré-testes com um número redu- 
zido de 6 participantes com perfil similar e 10 dias corridos de diário de uso. Os pré-testes ajudaram a aprimorar alguns pequenos problemas de configuração no Experience Fellow ${ }^{\circledR}$, relatar o mal funcionamento de alguns recursos aos desenvolvedores da plataforma e refinar os procedimentos de ativação remota de alguns convidados que não puderam começar os diários de uso conversando diretamente com o pesquisador responsável. É importante ressaltar que a única diferença da ativação remota para a presencial é que a remota consiste em explicações presentes por meio de um passo a passo com todo o processo do primeiro uso do Duolingo e Experience Fellow ${ }^{\circledast}$.

Ambas ativações começavam pelo teste de nivelamento do Duolingo de conhecimento da língua inglesa para delimitar o nível inicial de cada participante segundo a própria plataforma, com exceção do participante que possuía um Windowsphone, que não possui o teste de nivelamento e foi obrigado a começar do nível zero. Para os participantes que já conheciam o sistema e, portanto, poderiam já possuir uma conta, foi sugerida a criação de uma nova conta para demarcar a proficiência naquele momento. Feito o nivelamento, os participantes também precisavam habilitar seu cadastro na plataforma do Duolingo Escolar, para que os pesquisadores conseguissem acompanhar os estudos de cada um como se fossem um 'professor'.

Quanto à ativação do Experience Fellow ${ }^{\circledast}$ bastava o participante fotografar um QRCode através do app já instalado em seu dispositivo que o primeiro acesso o levaria para uma seção com os campos de nome, login do Duolingo, pontuação de nivelamento e telefone para eventual contato.

Com os participantes devidamente cadastrados em ambas as plataformas, foram estipulados 30 dias de uso para a pesquisa. Esse prazo foi definido devido ao tempo total do curso de inglês para falantes de português do Duolingo ser de apenas 36 horas. Tínhamos, então, 15 minutos diários de estudo (2 lições diárias), o que, no período estabelecido, representava aproximadamente 6 horas de exposições aos recursos de M-Learning. Um tempo expressivo de exposição para que o participante pudesse apresentar seu parecer sobre essa modalidade de aprendizagem móvel. $O$ app do Duolingo possui uma notificação automática diária informando a cada usuário que é necessário treinar o idioma. Além disso, semanalmente o pesquisador enviava um relatório das atividades feitas para cada um dos participantes pelo relatório do Duolingo Escolar.

Sempre que possível, o pesquisador tentava estimular os participantes a explorar o Duolingo indicando configurações ou outras funcionalidades da aplicação ou incentivando os participantes a tentar utilizar o app em diferentes 
lugares, como filas de banco, durante o almoço etc. O fator mobilidade era importante, pois o contexto múltiplo de uso do app Duolingo é um dos pontos fortes advogados pelo conceito de Aprendizagem móvel.

Uma questão importante delimitada para uma das etapas finais era a de que apenas os participantes que permanecessem na aplicação do Diário de uso por mais de um terço do período determinado, (mais de 10 dias de utilização) seriam convidados a fazer o teste de proficiência final, através do Duolingo Test Center.

Contudo, apesar de não fazer parte do objetivo geral da pesquisa verificar o progresso de aprendizado da língua inglesa dos participantes, a proposta era finalizar a pesquisa com um teste, para determinar o quanto os voluntários que foram expostos a mais de 2 horas de estudos na plataforma se sentiam confortáveis quanto ao conteúdo disponibilizado pelo app Duolingo e às exigências do teste de proficiência da própria plataforma, antes de participar da etapa final de debriefing.

\section{RESULTADOS E CONSIDERAÇÕES FINAIS}

Após o término de todos os Diários de uso com os participantes da pesquisa foi encerrado o acesso à plataforma Experience Fellow ${ }^{\circledR}$. Dos 15 participantes convocados, 5 deles desistiram antes de completar dez dias de pesquisa, portanto não efetuaram o teste de proficiência, precisando apenas participar, se possível, do debriefing de encerramento. Duas participantes [P14] e [P15] não participaram dessa última etapa, pois o pesquisador não conseguiu mais estabelecer contato formal com elas para finalização. Os motivos de desistência dos demais participantes foram expostos de forma mais detalhada na etapa do debriefing.

Na Figura 3 é possível ter uma visão geral das anotações feitas por cada um dos participantes por linha. A plataforma dispõe de algumas formas de classificação das anotações, sendo a da figura 3 uma visualização de acordo com a avaliação (ícones de humor) escolhida pelo participante ao escrever a nota, que podia variar de muito positivo $(+2)$ até muito negativo $(-1)$. A soma de todas as notas que os participantes deram ao app Duolingo, já contando com a inserção das notas do diário de papel do participante com o Windowsphone, foram um total de 263 anotações. 


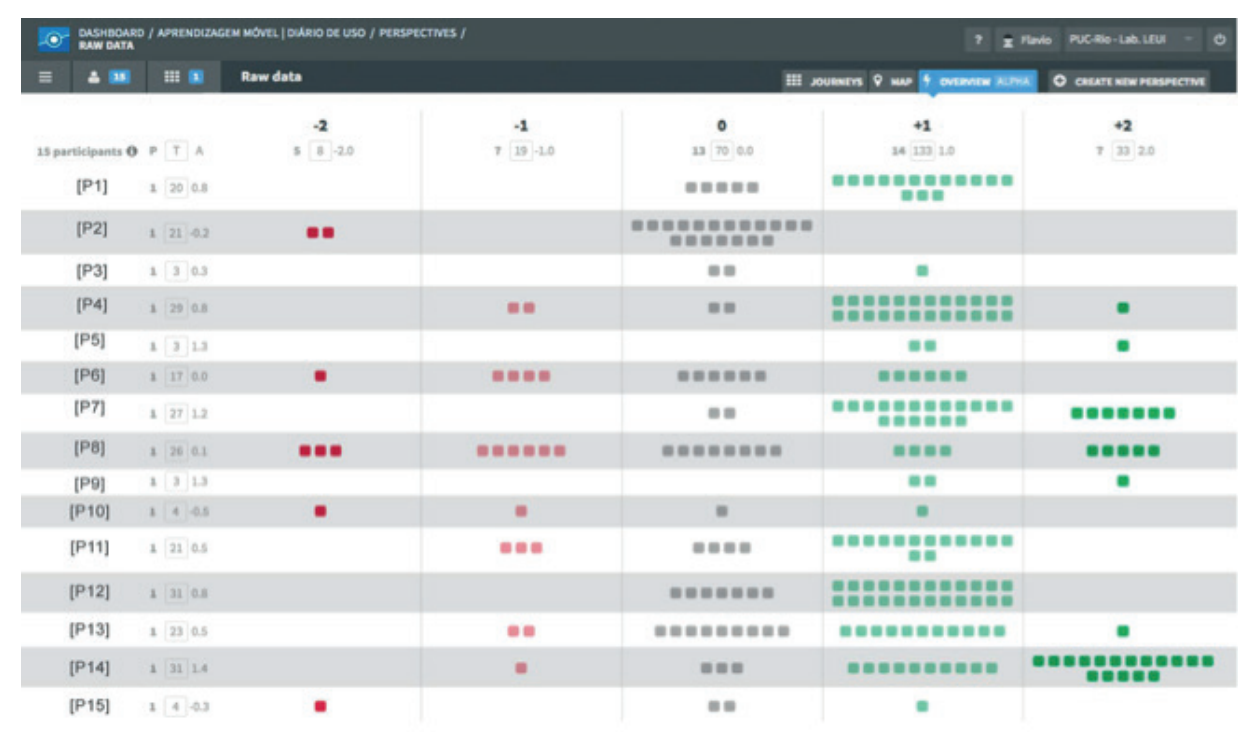

Figura 3: Experience Fellow - visão geral das anotações dos participantes Fonte: Website Experience Fellow, 2016.

Observando os detalhes de cada uma das anotações foi possível identificar que 37 das 263 anotações citam diretamente questões ligadas diretamente à usabilidade ou ao funcionamento técnico do produto. Por exemplo, verificou-se que a participante [P10] em uma de suas anotações mencionou que tinha pouco tempo para fazer os exercícios diários no Duolingo e elogiou a praticidade de utilizar o app no ponto de ônibus. Já o participante [P11] reportou uma falha de interação no template de exercício onde deve-se repetir em voz alta o áudio (speaking), indicando que o app "ouve" o próprio áudio do exercício caso o botão de falar seja pressionado simultaneamente ao botão de repetir a frase. Boa parte das anotações ligadas à Arquitetura de Informação e à Usabilidade dos participantes exaltam a satisfação quanto às questões ligadas a facilidade e velocidade do app no cotidiano, o que parece facilitar a inserção do Duolingo no cotidiano dos participantes. Quanto à insatisfação, foram citadas questões técnicas pouco claras na interface do usuário, como a possibilidade de pular exercícios de áudio quando não for o momento apropriado ou configurar as notificações e alertas do Duolingo para horários adequados ao usuário.

Os diários de uso digitais foram pertinentes para manter os participantes livres quanto a escolher em qual momento da sua rotina seria o ideal fazer uso deles. Portanto, as reflexões necessárias, as negociações, a complexidade da situação de cada uma das pessoas envolvidas, ao final contribuíram com o resultado de forma única para que cada um deles entendesse se o Duolingo fazia sentido nas suas vidas ou não.

A aplicação da técnica apresenta alguns desafios e parte deles foi reduzido atra- 
vés de pré-testes, como o recrutamento e a engajamento dos participantes ao longo do processo. Por exemplo, para manter o grupo coeso e ativo foi criado um grupo em um app de mensagem instantânea (whatsapp), onde dúvidas comuns entre os participantes, como a configuração de notificações ou outros recursos do app Duolingo ou Experience Fellow ${ }^{\circledR}$ poderiam ser tiradas. $O$ uso simultâneo de ambos aplicativos que poderia vir a ser um problema adicional, não gerou dificuldade no engajamento dos participantes, pois nenhum deles relatou problemas quanto à falta de tempo ou trabalho a mais. Mesmo a participante que utilizava um diário de papel se manteve anotando diariamente e mandando as fotos das páginas dos relatos, toda vez que fazia seus exercícios no Duolingo. Manter usuários engajados é um desafio à parte, considerando que o conteúdo disponibilizado pelo app Duolingo é muito básico para o conhecimento da maioria dos participantes. Ainda assim, ao final da pesquisa, boa parte dos participantes afirmaram que vislumbram a possibilidade da inserção em seus cotidianos de aplicativos de aprendizagem móvel, não necessariamente o Duolingo, mas outros com maiores desafios ou assuntos que poderiam ser explorados com mais profundidade.

Todavia a técnica de Diário de uso pode ser explorada com riqueza de detalhes para aferir hipóteses diversas e revela-se particularmente útil para um distanciamento interessante entre contexto de uso e acompanhamento do pesquisador.

\section{REFERÊNCIAS}

ALASZEWSKI, Alan. Using Diaries for Social Research. California: Sage, 2006. ALBERT, W. TULLIS, T. Measuring the User Experience: Collecting, Analyzing, and Presenting Usability Metrics. $2^{\circ}$ ed. Massachusetts: Morgan Kaufmann, 2013.

GOODMAN, E. KUNIAVSKY, M. MOED, A. Observing The User Experience. A Practitioner's Guide to User Research. California: Morgan Kaufmann, 2012. LAZAR, Jonathan; FENG, Jinjuan; HOCHHEISER, Harry. Research Methods in Human-Computer Interaction. CHichester: Wiley, 2010.

MATTELMÄKI, Tuuli. Design Probes. Publication Series of the University of Art and Design Helsinki. Vaajakoski, 2006.

MURPHY, J. Cultural probes: Understanding Users in Context. UXPAMagazine. Publicado em: Setembro 2006. Disponível em [http://uxpamagazine.org/ cultural_probes/]. Acesso em: 10 de Abril de 2016. 
NAZÁRIO, Flávio. MONT'ALVÃO, Cláudia. Da mochila à palma da mão: aprendizagem de línguas em contexto múltiplo - Um estudo sobre a usabilidade de aplicativo móvel de ensino de língua estrangeira. 2017. 255p. Dissertação de Mestrado - Dep. Artes \& Design, PUC-Rio. Rio de Janeiro, 2017.

SACCOL, A. SCHLEMMER, E. BARSOSA, J. M-Learning e U-Learning - novas perspectivas da aprendizagem móvel e ubíqua. São Paulo: Pearson Prentice Hall, 2011.

Cláudia Mont'Alvão: possui graduação em Desenho Industrial, Projeto de Produto, pelo Centro Universitário da Cidade (1994), mestrado e doutorado em Engenharia de Transportes pela Universidade Federal do Rio de Janeiro (1997 e 2001). Atualmente é Professora Associada/ Pesquisadora e Coordenadora do Programa de Pós Graduação em Design da Pontifícia Universidade Católica do Rio de Janeiro PPGDEsign PUC-Rio. Desde 2002 atua como Coordenadora do Laboratório de Ergodesign e Usabilidade de Interfaces (LEUI/PUC-Rlo). Como atividades decorrentes da atuação no LEUI/PUC-Rio é coordenadora geral dos eventos ERGODESIGN \& USIHC (eventos realizados desde 2000) e editora chefe da Revista Ergodesign \& $\mathrm{HCl}$ (lançada em 2013). Contribui ainda como consultora ad hoc de varias agências de fomento, tais como CNPq, FAPESP e FAPEMIG.

Tem como base de pesquisa o estudo do humano e o Ergodesign (a aplicação da Ergonomia aoDesign) em diversas áreas.

Desde 2012 suas pesquisas estao focadas em Educação a distância e mobile learning, E-health, $\mathrm{m}$-health plataformas dedicadas ao sistema de saúde, acessibilidade digital, Simbologia internacional/Compreensibilidade de pictogramas, e Design Gráfico no ambiente construído [Environmental GraphicDesign].

Flávio Nazário: Consultor de UX na empresa de tecnologia Concrete, colabora na estratégia das empresas que buscam inovação em produtos digitais. É Designer formado em Programação Visual e Projeto de Produto pela ESDI-UERJ, com especialização em Ergodesign e Mestrado em Design pela PUC-Rio. Trabalha desde 2010 na área de TI com Pesquisas de Inovação e Experiência do Usuário para diversos segmentos de mercado, desenvolvendo produtos/serviços para startups até multinacionais. Atualmente é pesquisador no Laboratório de Ergodesign e Usabilidade de Interfaces (LEUI - PUC-Rio) e contribui com iniciativas que fomentem comunidades de Design através do IxDA-Rio, além de auxiliar na organização de eventos e meetups sobre a sinergia entre Experiência do Usuário e Agilismo. 